\title{
VIOLENCIA CONTRA LA MUJER. UNA APROXIMACIÓN A SU DISMINUCIÓN DESDE LA ESCUELA SECUNDARIA.
}

\author{
Marilé Arias Méndez ${ }^{1}$ \\ Jacqueline García Pérez ${ }^{2}$
}

\begin{abstract}
Women and girls face various types of violence (physical, economic and psychological), ranging from sexual comments and whistling, fondling, and raping, to the most extreme form: femicides," as stated by the United Nations (UN) in a written communication. This worldwide organization called on governments, the private sector and society in general to eradicate the violence suffered by two out of three women; This particular problem in Mexico also shows alarming statistics which, in order to be visible nationwide, the States have issued violence alerts as a preventive measure.Education plays an important role in the transformation of this situation, rapists and rape victims are the result of educational processes that take
\end{abstract} place in classrooms, in the family and social contexts that have been organized about them, which obviously have not been completely efficient. In this regard, the intention is to organize clubs and work in favor of no gender-based violence since high school where standards and values are established, promoting women respect and consideration, and the demands of this level of education are maintained.

Keywords: Women and girls, types of violence, education, prevention, clubs.

\section{Introducción.}

$$
\text { En el marco del Día }
$$
Internacional de la Eliminación de la Violencia contra las Mujeres que es el 25 DE NOVIEMBRE y la campaña

\footnotetext{
${ }^{1}$ Profra. en Educación Primaria. Escuela Normal del Edo. de Tabasco "Rosario Ma. Gutiérrez Eskildsen", Lic. en Ciencias Naturales. Escuela Normal Superior "Justo Sierra Méndez" Puebla, Pue., Diplomado en Habilidades Docentes. Tecnológico de Monterrey, Maestría en Innovación Educativa. Universidad de los Ángeles, Campus Comalcalco, Tab., Doctorante en Excelencia Educativa. Universidad de los Ángeles, Campus Comalcalco, Tab.

${ }^{2}$ Lic. en Ciencias de la Educación. Universidad Juárez Autónoma de Tabasco, Maestría en Innovación Educativa. Universidad de los Ángeles, Campus Comalcalco, Tab., Doctorante en Excelencia Educativa. Universidad de los Ángeles, Campus Comalcalco, Tab.
} 
"ÚNETE" que tiene el objetivo de generar consciencia para prevenir y erradicar la violencia contra las mujeres y niñas.

La ONU de manera general y el área dedicada a estos temas detalló que, en México:

1. Nueve mujeres son asesinadas cada día en México.

2. Ocho de cada 10 se sienten inseguras y la mayoría ha sido agredida, seis de cada 10 niñas y adolescentes han sufrido al menos una forma de castigo psicológico o físico en sus hogares.

3. De 2015 a la fecha, suman 3,200 feminicidios a nivel nacional. Solo de enero a junio de 2019 se registraron 470 casos, según cifras del Secretariado Ejecutivo del Sistema Nacional de Seguridad Pública (SESNSP).

Las principales causas de estas formas de violencia son los estereotipos de género, la cultura machista, el patriarcado, la normalización y la naturalización de la violencia, y la minimización de su impacto. Todos estos factores estructurales perpetúan y fomentan la violencia contra las mujeres y niñas en el mundo y en México.

Bajo el lema de campaña "No dejes a nadie atrás: pon fin a la violencia contra las mujeres y las niñas", la ONU reforzó el llamado a hombres y a mujeres para fomentar una sociedad más igualitaria, ya que no se puede lograr un mundo donde nadie se quede atrás, cuando las mujeres, por el simple hecho de serlo, enfrentan de modo sistemático violencia y discriminación.

De acuerdo al INEGI los resultados de la Encuesta Nacional sobre la Dinámica de las Relaciones en los Hogares (ENDIREH) 2017, en México el 43.2 por ciento de las mujeres de 15 años y más sufrió algún incidente de violencia por parte de su pareja (esposo o pareja, ex-esposo o ex-pareja, o novio) durante su última relación. El porcentaje fluctúa entre 54.1 por ciento en el Estado de México y 33.7 por ciento en Coahuila de Zaragoza. De estas mujeres, 37.5 por ciento declaró haber recibido agresiones emocionales que afectaron su salud mental y psicológica; 23.4 por ciento recibió algún tipo de agresión para controlar sus ingresos y el flujo de los recursos monetarios del hogar, así como cuestionamientos con respecto a la forma en que gastaba dicho ingreso.

Dos de cada diez mujeres dijeron haber sufrido algún tipo de violencia física que les provocaron daños 
permanentes o temporales. Los contrastes por entidad federativa son notables: en Tabasco 25.7 por ciento de las mujeres vivió este tipo de violencia, y en Tamaulipas alcanzó 13 por ciento. Las mujeres víctimas de violencia sexual cometida por sus propias parejas representan 9 por ciento.

Como se puede apreciar, el estado de Tabasco, muestra indicadores desfavorables en este rubro de inclusión social, y aunque no muestra en la actualidad, una alerta de género, sí suceden diariamente actos de violencia contra la mujer.

\section{¿Por qué el tema de la violencia en México?}

En 2018, México alcanzó un récord histórico en feminicidio contra menores. En 2017, 86 niñas fueron asesinadas por violencia de género, la cifra más alta de la que se tenga registro. Mientras que, en total al año, se contabilizaron más de 800 casos de feminicidios en el país.

Con un panorama nacional de 14 estados con solicitudes de Alerta de Violencia de Género (AVG), y siete entidades más con AVG declaradas, el territorio mexicano se consolida como
212

una zona de riesgo para las mujeres, en una guerra que a diario cobra la vida de siete mujeres y otro tanto de desaparecidas, víctimas de trata y tortura sexual, agudizando la desigualdad estructural en la que viven las niñas, adolescentes y mujeres en México. Con base en el planteamiento anterior hay que reconocer que hace falta la incorporación de estos temas en las escuelas secundarias, como parte de la formación de niñas, adolescentes y jóvenes a manera de prevención.

\section{El Observatorio Ciudadano}

Nacional de Feminicidio (OCNF), monitorea y exige rendición de cuentas a las instituciones a cargo de prevenir y sancionar la violencia, registró de 2015 a junio de 2017, 4112 feminicidios en 13 estados. Un porcentaje fue cometido por uso excesivo de la fuerza física como golpes, contusiones, heridas con armas punzocortantes o asfixia. La mayor problemática de este tipo de violencia contra las mujeres es la impunidad y la violencia institucional de las autoridades quienes no investigan o no cuentan con protocolos de investigación, pericial, policial y ministerial con perspectiva de género y derechos humanos. 
Para 2016, los feminicidios se presentaron particularmente en 10 entidades donde se registra más violencia feminicida contra las mujeres: Chiapas, Chihuahua, Ciudad de México, Guerrero, Jalisco, Estado de México, Nuevo León, Oaxaca, Puebla y Sinaloa con un total de 1042 asesinatos de mujeres por razones de género.

Prevalecen feminicidios de mujeres/adolescentes/niñas que vivían en constante violencia, contextos estructurales en los que el Estado podría intervenir para prevenir la violencia feminicida, que afecta a mujeres de entre 15 y 35 años de edad.

En los estados con alta población indígena se registran más feminicidios contra mujeres de menos de 20 años. En zonas turísticas se presentan un importante número de feminicidios de mujeres extranjeras.

El estudio denominado Carga Global de la Violencia Armada 2015 "Cada Cuerpo Cuenta", señala que en seis años más de 1,900 mujeres y niñas fueron asesinadas de forma violenta en México y casi la mitad de ellas con armas de fuego, lo que nos da una clara relación entre la existencia de armas y la normalización de su uso en la violencia feminicida. Esto ubica al país entre los 10 con mayor número de feminicidios por armas de fuego en el mundo.

El Informe de la Comisión Nacional de Derechos Humanos (2017) revela que las entidades federativas con mayor número de averiguaciones previas iniciadas por el delito de trata son Puebla con 375, Chiapas 315, Ciudad de México 113, Oaxaca 118 y Baja California Norte y Estado de México con 95 cada una, lo cual representa el $76 \%$ del total iniciadas en el ámbito local.

De 2,843 víctimas identificadas en el Informe de la CNDH, $87.9 \%$ son mexicanas y solo $8.4 \%$ extranjeras; 93.4 $\%$ mujeres y $6.1 \%$ hombres; $26.5 \%$ son personas menores de edad y $71.7 \%$ mayores de edad $(3.2 \%$ no se identificó edad). El origen de las víctimas son los países: Estados Unidos, Honduras, Colombia, Argentina, El Salvador, Guatemala, Cuba, Chile, Nicaragua y Venezuela.

Los contextos de vulnerabilidad de las mujeres se han acrecentado por un conflicto armado y las estrategias de seguridad pública, ocasionando actos de extrema violencia contra las mujeres como la violencia sexual por parte de 
militares y grupos del crimen organizado.

El OCNF registró de enero de 2011 a junio de 2012, 3,976 mujeres y niñas desaparecidas en 15 estados, de éstos el $54 \%$ sucedieron en los estados de Chihuahua y Estado de México; en 51 $\%$ de los casos las mujeres tenían entre 11 y 20 años de edad.

A pesar de ello, la denuncia de desaparición de mujeres y niñas no es prioridad del Estado, mientras que el contexto de inseguridad ha provocado el incremento en la venta y explotación de mujeres.

Los elementos discutidos hasta aquí apuntan la necesaria toma de decisiones educativas, de orientación ciudadana para sus miembros, que posibilite el conocimiento del estado desfavorable de estos indicadores. Lo cual justifica la creación de un club en el contexto escolar a favor del tratamiento de estos temas en la escuela secundaria, aun cuando la Nueva Escuela Mexicana no contempla la implementación de clubes.

\section{Una discusión teórica sobre el tema.}

La Ley General de Acceso de las Mujeres a una Vida Libre de
214

Violencia (LGAMVLV), como parte del proceso de atención a las mujeres víctimas de violencia, creó el Banco Nacional de Datos e Información sobre Casos de Violencia contra las Mujeres, a cargo de la Secretaria de Seguridad Pública, que tiene como Objetivo General: "Proporcionar y administrar la información procesada de las instancias involucradas en la atención, prevención, sanción y erradicación de la violencia de género, con el fin de instrumentar políticas públicas desde la perspectiva de género y de derechos humanos de las mujeres", conforme a lo que reporta la Secretaría de Seguridad Pública.

Cuando un hecho delictivo derivó en una denuncia penal, como estas denuncias se realizan únicamente ante las instituciones encargadas de la procuración de justicia, el correspondiente registro de casos se encuentra en la Procuraduría General de Justicia de cada entidad federativa. Las Procuradurías Generales de Justicia reportan al Instituto Nacional de Estadística y Geografía (INEGI) información sobre ciertos presuntos delitos registrados en averiguaciones previas iniciadas por las agencias del ministerio público del fuero común. Esta 
información la reportan también al Secretariado Ejecutivo del Sistema Nacional de Seguridad Pública (SESNSP).

En México existen diferentes medios para la denuncia y conocimiento de los indicadores principales asociados al tratamiento de la violencia contra la mujer:

1. Encuesta Nacional sobre la Dinámica de las Relaciones en los Hogares, con dos levantamientos (2003 y 2006). En 2003 se explora la violencia de pareja en las mujeres de 15 años de edad y más, casadas o unidas. En 2006, el universo se amplía a todas las mujeres de 15 años y más, sin importar su estado conyugal; y se explora, además de la violencia de pareja, la violencia en otros ámbitos (laboral, escolar, comunitario, familiar y patrimonial). Los resultados del tercer levantamiento (2011) estarán disponibles a mediados del año 2012.

2. Encuesta Nacional sobre Violencia contra las Mujeres. Realizada por la Secretaría de Salud en dos levantamientos (2003 y 2006), en la que se tuvo como población objetivo a las mujeres usuarias de los servicios de salud. Un tercer levantamiento de esta encuesta está contemplado para el año 2012.

3. ¿Cómo educamos a nuestros/as hijos/as? Encuesta de Maltrato infantil y factores asociados 2006. Encuesta realizada por el INMUJERES y el Instituto Nacional de Psiquiatría Ramón de la Fuente Muñiz en 4 entidades federativas (Baja California, Sonora, Tlaxcala y Yucatán), con el objetivo de generar información estadística relacionada con el maltrato infantil y adolescente en México, en todas sus acepciones: maltrato físico, emocional, por abuso sexual y por descuido o negligencia.

4. Encuesta sobre la dinámica de las relaciones en el noviazgo entre las estudiantes de bachillerato y preparatoria de una escuela privada, 2006. Permite conocer la prevalencia de las diversas formas de violencia en las relaciones de noviazgo, la severidad de éstas y los agentes que intervienen para perpetuarlas: sociodemográficos, socioeconómicos, hábitos saludables, consumo de alcohol, tabaquismo, estupefacientes, ejercicio de la sexualidad, vida reproductiva, situación de pareja, violencia intrafamiliar, niveles 
de autonomía y poder de decisión de las mujeres e ideología de roles de género.

5. Encuesta Nacional sobre Violencia en el Noviazgo 2007. Encuesta realizada por la Secretaría de Educación Pública y el Instituto Mexicano de la Juventud, con el objetivo de identificar el contexto, magnitud, así como los factores determinantes de los diferentes tipos de violencia que se presentan en una relación de noviazgo; conocer la percepción de las y los jóvenes respecto de la violencia y de su manejo para la resolución de conflictos; así como cuantificar la prevalencia de la violencia que se presenta entre jóvenes, mujeres y hombres, entre 15 y 24 años de edad.

6. Informe Nacional sobre Violencia de Género en la Educación Básica en México 2008. Investigación realizada en un esfuerzo conjunto de la Secretaría de Educación Pública y el Fondo de las Naciones Unidas para la Infancia (UNICEF), con el propósito de generar conocimiento estadístico que permita una aproximación a la comprensión del fenómeno de la violencia de género en las escuelas para orientar el diseño y desarrollo de políticas educativas con enfoque de género y derechos de la infancia que propicien un mayor y mejor equilibrio entre los sexos, y que contribuyan de manera eficaz a garantizar los derechos de los niños y las niñas a la no discriminación.

Los principales indicadores que son contantemente estudiados relacionados con la violencia de la mujer son:

- Lesiones por consecuencia de violencia familiar por grupo de edad según sexo

- Mujeres casadas o unidas con violencia por parte de su pareja en los últimos 12 meses por tipo de violencia y tipo de localidad

- Mujeres casadas o unidas con violencia económica por parte de su pareja en los últimos 12 meses por grupos de edad

- $\quad$ Mujeres casadas o unidas con violencia emocional por parte de su pareja en los últimos 12 meses por grupos de edad

- Mujeres casadas o unidas con violencia física por parte de su pareja en los últimos 12 meses por grupos de edad

- $\quad$ Mujeres casadas o unidas con violencia sexual por parte de su 
pareja en los últimos 12 meses por grupos de edad

- Defunciones

por

homicidio según sexo

- $\quad$ Mujeres de 15 años y más

por tipo de violencia según ámbito

- Población penitenciaria del fuero común por situación jurídica según sexo

- Población penitenciaria del fuero federal por situación jurídica según sexo

- Población penitenciaria por fuero del delito según sexo

- $\quad$ Mujeres de 15 años y más de edad con algún evento de violencia de género en cualquier ámbito a lo largo de su vida por estado conyugal

- $\quad$ Presuntos homicidios por grupos de edad, según sexo y año de ocurrencia.

\section{El marco jurídico y los instrumentos}

de derechos humanos de las mujeres en el plano internacional.

Ya hace más de veinte años que las organizaciones de mujeres han venido legitimando la violencia basada en el género como un problema grave, creciente y de altos costos para los países y para las mujeres, de tal forma que ha
217

presionado a nivel nacional $\mathrm{e}$ internacional para que forme parte de las agendas de los gobiernos y del debate político.

A inicios de la década de los noventa la Organización Mundial de la Salud, OMS, el Banco Mundial y el Fondo de Población de las Naciones Unidas, FNUAP, concibieron a la violencia contra las mujeres como un problema de salud pública. Además, inicialmente se considera como un tema de derechos humanos de las mujeres en la Conferencia Mundial de Derechos Humanos de Viena, 1993, y en relación con la salud sexual y reproductiva en la Conferencia Internacional sobre Población y Desarrollo de El Cairo, 1994. Otro avance importante fue que la Plataforma para la Acción de la Cuarta Conferencia Mundial sobre la Mujer celebrada en Beijing, 1995, dedicó un capítulo completo a la violencia contra la mujer, dando como resultado una serie de recomendaciones para los gobiernos, las organizaciones multilaterales y no gubernamentales.

En ese sentido, en 1966, el Pacto Internacional de Derechos Civiles y Políticos estipula expresamente la prohibición de las discriminaciones por 
razón de sexo, y en el mismo año el Pacto Internacional de Derechos Económicos y Sociales de Naciones Unidas retoma expresamente esta prohibición al constatar que aun cuando estos instrumentos tienen eficacia vinculante para los Estados ratificantes, el garantizar su eficacia en términos de la discriminación por sexo distaba mucho de la situación ideal para las mujeres. Es así, como la Asamblea General de la ONU aprobó en 1979 la Convención sobre la Eliminación de todas las Formas de Discriminación Contra las Mujeres, CEDAW, la cual entro en vigor en 1981.

La CEDAW, define como discriminación de la mujer: "cualquier distinción, exclusión o restricción de sus derechos y libertades por el mero hecho de ser mujer". Este es un concepto esencial por cuanto las diferencia entre las mujeres respecto a otros grupos sociales en materia de derechos humanos con más vulnerabilidad, lo que se estipula como conocemos violencia de género o discriminación de género, consiste en que la discriminación o la violencia contra las mujeres se produce generalmente por el solo hecho de ser mujeres, no por lo que hayan hecho o dejado de hacer. Entre los derechos a no sufrir exclusión o restricción de sus derechos y libertades por ser mujer, se establece que ese derecho debe ser la guía de la acción política de los poderes de los Estados.

Se establece también, el principio de igualdad entre el hombre y la mujer, pero se aclara la necesidad de que la igualdad entre los géneros se traduzca en consecuencias prácticas, lo que no venía sucediendo en su totalidad.

\section{¿Qué es la violencia contra la mujer?}

La Declaración sobre la Eliminación de la Violencia contra la Mujer, aprobada en Resolución de la Asamblea General de Naciones Unidas 48/104, del 20 de diciembre de 1993, publicada el 23 de febrero de 1994 (ONU,1993) es el primer documento a nivel internacional, que aborda de manera clara y específica esta tipología de violencia, definiendo en su artículo primero que la violencia contra la mujer es todo acto de violencia basado en la pertenencia al sexo femenino, que tenga o pueda tener como resultado un daño o sufrimiento físico, sexual o psicológico para la mujer, así como las amenazas de tales actos, la coacción o la privación arbitraria de la libertad, tanto si se 
produce en la vida pública como en la vida privada.

De acuerdo con la Organización de las Naciones Unidas, el término se utiliza "para distinguir la violencia común de aquella que se dirige a individuos o grupos sobre la base de su género", constituyéndose en una violación de los derechos humanos; tal enfoque es compartido por Human Rights Watch en diversos estudios realizados durante los últimos años.

La violencia de género presenta distintas manifestaciones, como actos que causan sufrimiento o daño, amenazas, coerción u otra privación de libertades. Estos actos se manifiestan en todos los ámbitos de la vida social y política, entre los que se encuentran la propia familia, el Estado, la educación, los medios de comunicación, las religiones, el mundo del trabajo, la sexualidad, las organizaciones sociales, la convivencia en espacios públicos, la cultura, etc.

Existe consenso respecto a que la mayoría de las víctimas de la violencia de género son mujeres y niñas, mientras que históricamente los varones estarían subrepresentados en las estadísticas; otros estudios afirman que la población
LGBT también estaría subrepresentada e incluso, algunos grupos mostrarían mayor prevalencia a nivel comparado. ONU Mujeres advierte sobre el error habitual de considerar la expresión «violencia de género» como sinónima de la expresión «violencia contra la mujer», señalando que el concepto de violencia de género es más amplio y tiene como fin destacar la dimensión de género en la subordinación de la mujer en la sociedad y su vulnerabilidad frente a la violencia, que es dirigida contra cualquier persona que no respete los roles que una sociedad determinada le impone a hombres y mujeres, razón por la cual también hombres y niños pueden ser víctimas de la violencia de género, especialmente de la violencia sexual.

Por otra parte, la violencia de género es la violencia que ejerce alguien sobre una persona sólo por su género. Las acciones que consideramos violentas son todas aquellas que afectan de forma negativa a la identidad, la sexualidad y libertad reproductiva, la salud física y mental y el bienestar social de una persona.

El concepto de violencia de género, en algunas legislaciones, se reduce a la violencia ejercida hacia las 
mujeres, pero es más complejo que eso.

Este tipo de violencia incluye dinámicas de dominación, incluyendo las amenazas y la privación arbitraria de las libertades políticas y civiles, independientemente del ámbito en el que sucedan.

Entre los actos más aberrantes de violencia de género podemos encontrar ablación de clítoris, el infanticidio femenino, las violaciones, la prostitución forzada, los abortos en función del sexo del no nato, la violencia contra las prostitutas, la castración, el tráfico de personas, el acoso $\mathrm{y}$ hostigamiento dentro de organizaciones, y ataques homolesbofóbicos.

Según esta misma fuente, los tipos de violencia más frecuentes son:

1. Física: este tipo de violencia implica un accionar que produzca dolor y lesiones, o que tenga riesgo de producirlos. Este tipo de agresión afecta la integridad física de la persona. Puede darse en relaciones laborales, personales o familiares por igual y variar desde empujón hasta un intento de homicidio. Este tipo de maltrato es el que resulta más sencillo de demostrar en una corte.

2. Psicológica: los actos de violencia psicológica son todos aquellos
220

que producen sensaciones de desvalorización y sufrimiento. Estos actos pueden ir desde insultos, a actitudes extremas de control y reproches, humillaciones, autoridad, falta de respeto, imponer un aislamiento o incomunicación, entre otros. Si bien sus secuelas son difíciles de detectar, sus efectos a largo plazo pueden afectar la estabilidad mental y emocional de la víctima.

3. Sexual: en este caso las acciones que vulneren la libertad de elegir cuando, como y de qué manera tener relaciones sexuales serán denominadas violencia sexual. Aún si no se vulnera esta libertad por la fuerza, si no con amenazas o chantajes e intimidación. La violencia sexual incluye violaciones, abusos e interferencias en las elecciones reproductivas.

4. Económica: las acciones que impliquen la retención, destrucción o sustracción de bienes o dinero ilegítimamente por parte del victimario serán denominadas violencia económica. Este tipo de agresión produce la disminución o la eliminación total del bienestar físico y mental de la víctima y sus hijos. 
5. Simbólica: este tipo de opresión se ejerce a través de mensajes, medios de comunicación y estereotipos de fuercen a alguien a tomar una posición inferior en relación a otro género.

6. Doméstica: un hecho violento es considerado doméstico si lo realiza un integrante del grupo familiar, no en necesario convivir con el agresor. Dentro del grupo familiar podemos encontrar a parientes sanguíneos y a miembros por afinidad como una pareja.

\section{¿Qué son los clubes en el contexto de} las escuelas secundarias?

En el sexenio de Enrique Peña Nieto, una de las grandes trasformaciones que potenciaba el nuevo modelo educativo lo era precisamente el indicador de autonomía curricular, el cual pretendía entre otros aspectos, la posibilidad que tiene la escuela y las instituciones educativas a realizar incorporaciones curriculares y ajustes a favor de una mejor formación integral de sus educandos. Lo cual fue un acierto porque los contextos en los que están ubicadas las escuelas no son iguales por lo que cada quien de acuerdo a sus necesidades podía modificar o ajustar, por lo que se considera un acierto que en la Nueva Escuela Mexicana para este ciclo escolar (2019-2020), se dejara la decisión al Consejo Técnico Escolar de continuar con los clubes

\section{La Autonomía Curricular} responde a los principios de equidad e inclusión, además de que facilita la convivencia de estudiantes de grados y edades diversas; reorganiza al alumnado por habilidad o interés, y considera horas que cada plantel tenga disponibles. La Autonomía Curricular, que no requerirá mayor inversión de recursos, tiene cinco ámbitos:

\section{- Ampliar la formación} académica: estrategias de aprendizaje y técnicas de estudio; lenguaje $\mathrm{y}$ comunicación, con taller de escritura creativa, inglés y debates; taller de matemáticas; taller de tecnología, y taller de exploración de condiciones del medio y cambio climático.

- Potenciar el desarrollo personal y social: ligas deportivas, orquestas escolares, y talleres de teatro, danza, pintura, y convivencia escolar.

- Nuevos contenidos relevantes: educación financiera, programación y robótica, y emprendimiento. 


\section{- $\quad$ Conocimientos}

regionales, con microhistoria; taller de tecnología y artesanías locales; cultivo, hortalizas y plantas medicinales de la localidad; educación ambiental contextualizada, y lenguas originarias.

- Proyecto de impacto social, con limpieza de basura; potabilización del agua; cuidado de la salud; huertos y elaboración de composta, y herbolaria.

$$
\text { Relacionado con estos }
$$

elementos descritos en los espacios anteriores se define el club, como el espacio creado en el contexto de las nuevas transformaciones de la educación en México que potencia el desarrollo integral de los niños adolescentes y jóvenes contemplado en los horarios docentes y que para su definición deberá responder a ciertas exigencias descritas en los documentos metodológicos de la escuela o institución educativa.

En agosto del 2019 se realizaron talleres para tener un acercamiento a lo que será la Nueva Escuela Mexicana, en la que se promoverá una cultura de género que transforme los estereotipos del rol social de mujeres y hombres. No se trata sólo de corregir prejuicios, sino de erradicar convencionalismos que sesgan ra auténtica convivencia y coexistencia de ambos géneros.

Si bien es cierto que los padres de familia son responsables de la formación de los adolescentes y jóvenes también es cierto que la educación formal juega un papel importante en la transformación en la que maestras y maestros son responsables del proceso educativo que contribuye a la formación de sus alumnos $\mathrm{y}$, por lo tanto, se reconoce su contribución a la trasformación social, en el caso específico del tema tratado en este artículo, a la prevención de la violencia de género, disminuyendo o erradicando la cultura del machismo causa de los feminicidios y maltratos a mujeres, de esta manera se cumplirá lo establecido en el artículo $3^{\circ}$ Constitucional que señala que la educación se basará en el respeto irrestricto de la dignidad de las personas, con un enfoque de derechos humanos y de igualdad sustantiva.

En tal sentido se pretende organizar clubes y trabajar en el tratamiento a favor de la no violencia de género desde la escuela secundaria donde se establecen normas y valores a favor del respeto y la consideración de la 
mujer, sosteniendo las exigencias de este nivel de enseñanza.

\section{Bibliografía}

CONAPO, Indicadores demográfico 1990-2030.

CONAVIM, Análisis y evaluación de la política de acceso de las mujeres a una vida.

Libre de violencia implementado por el gobierno mexicano (2000-2009), México, SEGOB, 2010.

CONAVIM, Consultoría para el desarrollo de estrategias de atención, prevención y erradicación de la violencia contra las mujeres, México, SEGOB, 2009.

GDF, Cuarto informe de Trabajo

Secretaría de Desarrollo Social, México, 10 de octubre de 2010.

GDF, Carpetas de Informe del Consejo para la Asistencia y Prevención de la Violencia Familiar en el Distrito Federal, 2009 y 2010.
INEGI, Encuesta Nacional sobre la

Dinámica de las Relaciones en los Hogares 2003.

ENDIREH. Estados Unidos Mexicanos, México, INEGI, 2003.

INEGI, Conteo de Población y Vivienda 2005, México, INEGI, 2005.

INEGI, Encuesta Nacional sobre la Dinámica de las Relaciones en los Hogares 2006.

ENDIREH. Tabulados básicos. Estados Unidos Mexicanos, México, INEGI, 2008.

"Este programa es público ajeno a cualquier partido político. Queda prohibido el uso para fines distintos al desarrollo social". 97

INEGI, "XII Censo de Población y Vivienda 2000, Tabulados básicos" en: INEGI,

Mujeres y hombres en México, México, INEGI, 2009.

INMUJERES, Legislar Con Perspectiva de Género: Distrito Federal, México. 
Martínez Rodríguez Laura y Miriam

Valdez Valerio, Violencia de Género.

Visibilizando lo invisible, ADIVAC-

Indesol, México, 2007.

Organización de las Naciones Unidas, Informe de México producido por el Comité para la Eliminación de la Discriminación contra la Mujer bajo el Artículo 8 del Protocolo Facultativo de la Convención y respuesta del Gobierno de México, 27 de enero de 2005.

Organización de las Naciones Unidas, E/CN.4/2006/61/Add.4 Informe de la Relatora Especial sobre la violencia contra la mujer, sus causas y consecuencias, Yakin Ertürk sobre su misión a México (21 a 25 de febrero de 2005), 13 de enero de 2006.

Tarjetas municipales y estatales del sistema de Indicadores de Género del INMUJERES:

http://estadistica.inmujeres.gob.mx/for mas/index.php.

https://politica.expansion.mx/mexico/20 19/08/19/datos-sobre-violencia-contramujeres-mexico http://concepto.de/violencia-degenero/\#ixzz58zJO3Fx2 